\title{
Opportunities and Limitations in the Study of Transient Takotsubo Syndrome in Animal Models
}

\author{
Paolo Angelini ${ }^{\mathrm{a}, \mathrm{c}}$, Maria T. Gamero ${ }^{\mathrm{b}}$
}

Over the last several years after its initial recognition [1], some attempts have been made to artificially reproduce transient takotsubo syndrome (TTS), a complex and persistently unknown human pathology, by using animal models [2-5]. But, to make experiments be meaningful, can any of these attempts reproduce the essential and distinguishing features of human TTS? This update of the current discussion attempts to clarify what this new entity consists of, what published early experiments meant, and then to suggest new animal models that could better duplicate similar dysfunctional behavior.

Firstly, it is important to emphasize that indeed, experimental animal models can potentially be key tools for investigating a novel and obscure human disease, but only if some distinctive features of the model faithfully correspond to those of the human disease (its nature, its pathophysiological mechanisms, its appearance on imaging, and its clinical course). When dealing with a model of a human disease that is still nebulous in its essence and that has a wide spectrum of clinical presentations, it is important to start by carefully describing our best current understanding of the disease under study. Attempts to develop relevant animal models should initially focus on basic anomalies in clinical presentations and reproduce similar phenotypic features.

\section{What is Transient Takotsubo Cardiomyopathy?}

Summarizing a current characterization of the essential features of TTS, we propose the following items [6-8].

Human TTS is most frequently characterized by de novo, sudden-onset transient apical ballooning (dyskinesia), usually affecting $35-50 \%$ of the left ventricle (LV) (causing the ejection fraction to drop from $60-70 \%$ to $20-50 \%$ ) and accompanied simultaneously by hyperfunction of the remnant segments of the LV. Most cases (99\%) involve a discrete symmetrical

Manuscript submitted May 18, 2020, accepted May 22, 2020

Published online June 4, 2020

aDepartment of Cardiology, Texas Heart Institute, Houston, TX, USA

${ }^{b}$ Department of Medicine, Universidad Peruana Cayetano Heredia, Lima, Peru ${ }^{\mathrm{c} C}$ Corresponding Author: Paolo Angelini, Department of Cardiology, Texas Heart Institute, 6624 Fannin Street, Suite 2780, Houston, Texas 77030, USA.

Email: pangelini@leachmancardiology.com

doi: https://doi.org/10.14740/jocmr4225 (circumferential) territory along the longitudinal axis of the LV, independent of any single coronary branch territory. Additionally, identifying TTS forms usually requires that no critical coronary atherosclerotic stenosis be recognized by angiography (that could explain the LV dysfunction) [6-9].

The typical clinical history of TTS includes some variable and nonspecific human conditions, events, or morbid insults that may have triggered its onset, but many different clinical conditions can coexist with TTS and may or may not be intrinsic to its genesis; also, in $30-40 \%$ of cases, patients have no recognized pre-existent condition [6-9]. Typically, the events that are claimed to precede/precipitate TTS episodes occurred on multiple prior occasions before a given TTS episode or were chronic, and they may eventually worsen during TTS (like stress) or reoccur after the episode, without concomitant TTS recurrence [6-9].

TTS resolves spontaneously and completely over a few days in $90 \%$ of cases [6-9], and mostly it occurs only once in a person's lifetime (enabling what seems a sort of "automatic vaccination," rarely previously observed in medicine) [10].

Temporary, evolutionary and transient ST-T electrocardiographic changes usually occur [5-9]. Q-wave changes rarely appear.

Commonly, inconsistency is reported between the extent of the initial area of LV akinesia or dyskinesia (the apparent extent of the myocardial injury) and the peak elevation of serum cardiac enzyme levels, in comparison to an equivalent area of a typical acute myocardial infarction [10, 11].

The usual end-result of a TTS event is essentially a quick return of intact regional myocardial function in a few days in the absence of any recanalization intervention, suggesting that TTS is a myocardial stunning episode (temporary dysfunction that does not cause permanent scarring [6-9].

Hospital mortality is relatively low (about 2-5\%); however, it is likely that mortality mainly occurs during the first hour(s) after the onset of symptoms, usually in an extra-hospital environment, and therefore is significantly underreported [11-13]. This notion is supported by the fact that autopsy studies commonly do not reveal myocardial histologic changes typical of myocardial infarction $[11,12]$.

Although several claims have been made in the literature about the frequent occurrence of triggering or causative factors at the onset of TTS [12], recurrent and reliable causes of TTS have not been identified ("anything goes" [14]). One putative cause of TTS is stress (hence the term "stress cardiomyopathy"). The physiological correlates of stress have never 


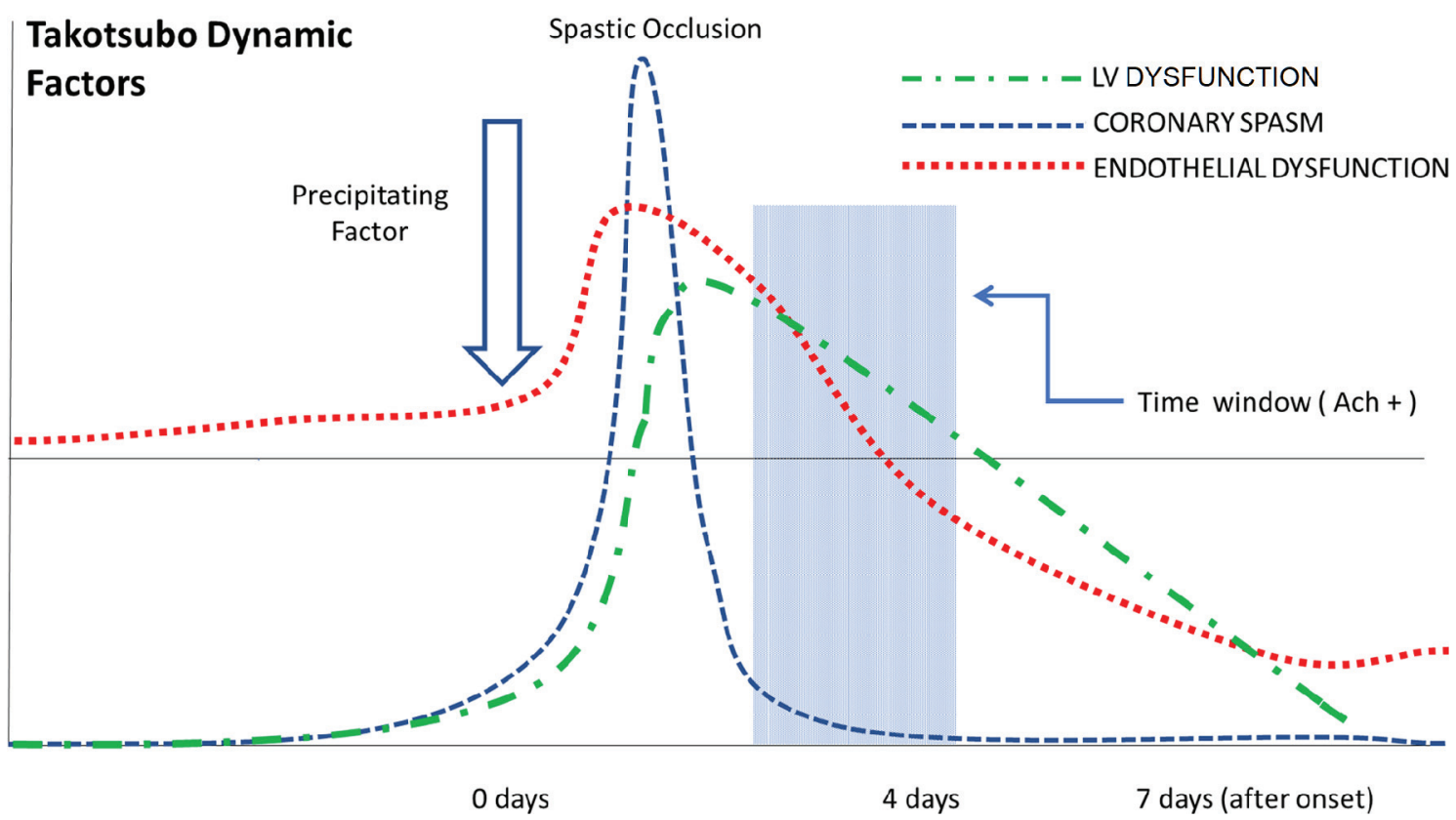

Figure 1. Diagrammatic representation of the dynamic factors involved in the appearance and disappearance of TTS features. The red line represents the variable state of endothelial dysfunction (ED); an episode starts with borderline ED that worsens in response to some precipitating factor, and then recovers gradually over $1-2$ days. The blue line indicates the appearance of severe epicardial coronary arterial spasm, which quickly resolves, while left ventricular dysfunction appears. Spastic transient occlusion must resolve in $15-60 \mathrm{~min}$ if no thrombosis occurs (which is rare). The blue area represents the time course of positive Ach testing (days 2 - 5). Usually, ED recovers to within normal limits on about day 5 (i.e., at the time of negative Ach testing) [20]. TTS: transient takotsubo syndrome.

been quantified by any measurable method. Mental upsets are frequent in the life of any human being, but the association between the severity of such stress and catecholamine levels (an elevated serum level is the claimed mechanism), initially claimed by Wittstein and colleagues [15], has never been confirmed. Currently, catecholamine serum levels (a potential measure of stress severity) are rarely measured in clinical settings, evidence that this marker is no longer considered clinically relevant, but "catecholamine cardiomyopathy" is another term in popular use, even though the concept is unconfirmed.

The so-called catecholamine surge has been frequently assumed but rarely demonstrated, and no prospective studies have ever measured catecholamine levels at the onset of an episode of TTS. Nor have any studies used this theory to reproduce TTS experimentally in humans by elevating serum catecholamine levels $[8,16]$.

Chronic catecholamine cardiomyopathy in pheochromocytoma $[8,16,17]$, the ultimate example of a spontaneous, long-term catecholamine overload with recurrent peaks, presents clinically with recurrent episodes of chest pressure, pounding of the heart, sinus tachycardia, hypertension, and an electrocardiographic LV strain pattern $[16,17]$. In this scenario, diffuse hypertrophy and progressive chronic scarring of the myocardium are common, as shown by magnetic resonance imaging or histologic examination $[16,17]$.

In some TTS cases, experimental acetylcholine (Ach) testing for endothelial dysfunction (done shortly after an event) has been reported to reproduce chest pain, ST changes, apical dyskinesia, and diffuse and transient regional coronary spasm with coronary arteriography. Left ventricular echocardiographic changes also occur and are similar to those observed in the original TTS episode $[18,19]$. Apical dyskinesia and coronary spasm are quickly and completely resolved by intracoronary nitroglycerine $[18,19]$.

Thus, the Ach test is recognized by some (albeit few) investigators as the only way to reproduce TTS in human beings, and it could or should be used to test the theory that TTS is actually the product of many alternative triggering stimuli in patients who are transiently affected by endothelial dysfunction and a spontaneous tendency to coronary spasm [18].

It is not currently known whether any animal species exists in which human TTS can be replicated during Ach testing, but this can be potentially tested, for example, in animals treated with catecholamines after endothelial dysfunction is induced by chemotherapeutic agents, or by radiation to the heart region or acute brain pathology (Fig. 1) [20].

Also, Ach testing provides objective evidence for using calcium antagonists and nitrates, ideally at the very onset of TTS (as is typically done in cases with recurrent TTS), to abort a full episode.

Unfortunately, this hypothesis (spasm-related pathophysiology) seems to be foreign to most cardiologists at present. The frequently raised objection is, "If the spastic pathogenesis of TTS is truly systematically involved in clinical TTS, why is the test not more frequently positive?" This question is addressed by our group's hypothesis that the underlying cause of the positive Ach test rapidly dissipates after an episode of TTS (consistent with the rarity of clinical TTS recurrence when 
done late after an event and the auto-vaccination concept described above) [20].

In our own experience, those rare patients who have a positive Ach test more than 1 week after a TTS episode have a high rate of TTS recurrence (or of Prinzmetal angina) [10, 18].

Unfortunately, most of the rare reports of Ach (or ergonovine) testing in TTS do not include the time of testing since TTS onset, or the dose, or the reason for testing, which are fundamental limitations on interpreting the results of testing.

From our group's admittedly limited experience in this field, we are inclined to conclude that the best window of time for eliciting a positive response to Ach is between less than 1 day (when the probability of a positive test is potentially $100 \%$, but the patient is unstable at that early time, when the worst level of cardiomyopathy is present) to 7 days (when the probability of positive testing is less than $10 \%$ ) after the onset of TTS $[18,20]$.

\section{Animal Models of Experimental Takotsubo?}

As a consequence of our persistent ignorance regarding TTS etiopathogenesis, some investigators have already attempted in recent years to duplicate some or all of the abovementioned clinical manifestations of TTS by using animal models treated with high doses of catecholamines or agonist drugs [2-4]. In studies involving basic animal catecholamine experiments, mortality is high (30-80\%) [2-5], suggesting that the doses used are toxic to the point of being frequently lethal $[21,22]$. Clearly, this is a very different mortality rate than that associated with clinical TTS, as stated above.

From the onset, one should ask a critical question: Are elevated catecholamine levels the true cause of human TTS, or are they only a triggering factor in some patients with endothelial dysfunction (transient abnormal vasospastic hyperreactivity that can be demonstrated by Ach administration)? To date, animal experiments have never produced imaging proving localized and transient myocardial ballooning dysfunction (symmetrical dyskinesia, which is rapidly reversible) and thus cannot be considered proof of TTS reproduction in one of its basic manifestations [21].

At the same time, it is relevant to recognize in reviewing clinical cases featuring high levels of catecholamines (i.e., pheochromocytoma, paraganglioma), a sort of natural experiment in a human subject that TTS is rarely observed in the years, when patients are treated medically, before or after surgical treatment $[16,17,22]$. Evidently, only some patients (candidates for a TTS diagnosis), and only for some portion of their lives, develop endothelial dysfunction associated with high catecholamine levels.

These recent animal experiments instead seem to simulate a different pathology from TTS in pheochromocytoma. The resulting cardiomyopathy more commonly seen in human pheochromocytoma (which is chronic and only partially reversible, and accompanied by diffuse fibrotic degeneration $[17,22]$ ) is not TTS-related and has an essentially different prognosis (chronically progressive and generally irreversible cardiomyopathy).

Moreover, the clinical use of inotropic agents (i.e., dobu- tamine) in stress testing, or to treat hypotension or shock, only rarely triggers TTS [18]. A recent meta-analysis [8], inspired by the controversy over the etiology of TTS, rebutted the theory of Wittstein et al [15], suggesting instead that the catecholamine surge is not the isolated cause, but may be more of a trigger of TTS (whereby catecholamines would be not the minimum common denominator, but a variable, nonessential precipitating or triggering factor).

Additionally, specific human clinical study showed recently that serum catecholamine levels in stressful human situations are only 2 - 3 times their baseline levels, much less than the amounts used in experimental animal models [23].

Interestingly, the association occasionally observed between TTS and acute pathology of the central nervous system has led some to suggest the term "neurogenic stunned myocardium," which could be very suggestive of TTS [18]. Pilot studies in animals have been initiated that may suggest effective reproduction of transient cardiomyopathy in this context [24].

Finally, we would propose that studies of segmental wallmotion abnormalities use larger animals than rats or mice, whose heart is quite small and beats too rapidly for reliable echocardiographic evaluation (about 300 beats/min at rest, and up to 600 beats/min after a large catecholamine dose) [12].

In conclusion, we should agree that to date, animal models of TTS have been inadequate in terms of replicating the distinguishing features of human TTS and in clarifying the persistently mysterious etiopathogenesis of its human presentations. However, improvements are possible, especially by inducing endothelial dysfunction in animals before administering catecholamines. Such experiments could add to the direct evidence related to TTS, providing an experimental basis for "endothelial dysfunction," still a somewhat vague entity that spontaneously and mysteriously disappears after a TTS episode $[25,26]$. The possibility that TTS is the product of microvascular pathology $[13,19]$ remains an unlikely hypothesis without a solid anatomically and physiologically demonstrable basis. Incidentally, positive Ach testings in humans show severe spastic behavior in epicardial segments but also in the smallest coronary arteries (arterioles, probably in the range of $0.5 \mathrm{~mm}$ diameter), that are currently visible by catheter angiography [18].

In conclusion, our hypothesis is that human TTS is usually the product of both pre-existent endothelial dysfunction (increasing during a spell) and a variable catecholamine surge or stress (precipitating a facultative event). This knowledge creates an opportunity to develop better animal models than the standard standalone catecholamine-based ones.

\section{Acknowledgments}

We would like to thank Stephen N. Palmer, PhD, ELS, who expertly contributed to the editing of the manuscript.

\section{Financial Disclosure}

None to declare. 


\section{Conflict of Interest}

None to declare.

\section{Author Contributions}

Paolo Angelini contributed to conceptual development and text editing; Maria T. Gamero contributed to writing and literature search.

\section{Data Availability}

The data supporting the findings of this study are available from the corresponding author upon reasonable request. Any inquiries regarding supporting data availability of this study should be directed to the corresponding author.

\section{References}

1. Dote K, Sato H, Tateishi H, Uchida T, Ishihara M. [Myocardial stunning due to simultaneous multivessel coronary spasms: a review of 5 cases]. J Cardiol. 1991;21(2):203214.

2. Ali A, Redfors B, Lundgren J, Alkhoury J, Oras J, Gan LM, Omerovic E. Effects of pretreatment with cardiostimulants and beta-blockers on isoprenaline-induced takotsubo-like cardiac dysfunction in rats. Int J Cardiol. 2019;281:99-104.

3. Redfors B, Ali A, Shao Y, Lundgren J, Gan LM, Omerovic E. Different catecholamines induce different patterns of takotsubo-like cardiac dysfunction in an apparently afterload dependent manner. Int J Cardiol. 2014;174(2):330-336.

4. Sachdeva J, Dai W, Kloner RA. Functional and histological assessment of an experimental model of Takotsubo's cardiomyopathy. J Am Heart Assoc. 2014;3(3):e000921.

5. Shao Y, Redfors B, Scharin Tang M, Mollmann H, Troidl C, Szardien S, Hamm C, et al. Novel rat model reveals important roles of beta-adrenoreceptors in stress-induced cardiomyopathy. Int J Cardiol. 2013;168(3):1943-1950.

6. Napp LC, Bauersachs J. Takotsubo syndrome: between evidence, myths, and misunderstandings. Herz. 2020;45(3):252-266.

7. Templin C, Ghadri JR, Diekmann J, Napp LC, Bataiosu DR, Jaguszewski M, Cammann VL, et al. Clinical features and outcomes of takotsubo (stress) cardiomyopathy. N Engl J Med. 2015;373(10):929-938.

8. S YH, Tornvall P. Epidemiology, pathogenesis, and management of takotsubo syndrome. Clin Auton Res. 2018;28(1):53-65.

9. Bybee KA, Kara T, Prasad A, Lerman A, Barsness GW, Wright RS, Rihal CS. Systematic review: transient left ventricular apical ballooning: a syndrome that mimics ST-segment elevation myocardial infarction. Ann Intern Med. 2004;141(11):858-865.

10. Angelini P. Recurrent takotsubo cardiomyopathy: an op- portunity to clarify causation and prognosis. Tex Heart Inst J. 2018;45(4):252-253.

11. Angelini P. Do pathologists agree on how to diagnose takotsubo cardiomyopathy? Forensic Sci Med Pathol. 2016;12(2):226.

12. Angelini P, Uribe C. Cardiac arrest in takotsubo cardiomyopathy. Am J Cardiol. 2015;116(3):489-490.

13. Lyon AR, Bossone E, Schneider B, Sechtem U, Citro R, Underwood SR, Sheppard MN, et al. Current state of knowledge on Takotsubo syndrome: a position Statement from the taskforce on takotsubo syndrome of the Heart Failure Association of the European Society of Cardiology. Eur J Heart Fail. 2016;18(1):8-27.

14. Angelini P. Tako Tsubo, where everything can be variable: or can it? The quest for solid grounds in a slippery entity. Europace. 2008;10(12):1359-1360.

15. Wittstein IS, Thiemann DR, Lima JA, Baughman KL, Schulman SP, Gerstenblith G, Wu KC, et al. Neurohumoral features of myocardial stunning due to sudden emotional stress. N Engl J Med. 2005;352(6):539-548.

16. S YH. Plasma epinephrine level and its causal link to takotsubo syndrome revisited: critical review with a diverse conclusion. Cardiovasc Revasc Med. 2019;20(10):907914.

17. Batisse-Lignier M, Pereira B, Motreff P, Pierrard R, Burnot C, Vorilhon C, Maqdasy S, et al. Acute and chronic pheochromocytoma-induced cardiomyopathies: different prognoses? A systematic analytical review. Medicine (Baltimore). 2015;94(50):e2198.

18. Angelini P. Transient left ventricular apical ballooning: A unifying pathophysiologic theory at the edge of Prinzmetal angina. Catheter Cardiovasc Interv. 2008;71(3):342352.

19. Verna E, Provasoli S, Ghiringhelli S, Morandi F, SalernoUriarte J. Abnormal coronary vasoreactivity in transient left ventricular apical ballooning (tako-tsubo) syndrome. Int J Cardiol. 2018;250:4-10.

20. Angelini P, Uribe C. Is transient takotsubo syndrome associated with cancer? Why, and with what implications for oncocardiology? J Am Heart Assoc. 2019;8(15):e013201.

21. Angelini P, Tobis JM. Is high-dose catecholamine administration in small animals an appropriate model for takotsubo syndrome? Circ J. 2015;79(4):897.

22. Gagnon N, Mansour S, Bitton Y, Bourdeau I. Takotsubolike cardiomyopathy in a large cohort of patients with pheochromocytoma and paraganglioma. Endocr Pract. 2017;23(10):1178-1192.

23. Dimsdale JE, Moss J. Plasma catecholamines in stress and exercise. JAMA. 1980;243(4):340-342.

24. Koza Y, Aydin N, Aydin MD, Koza EA, Bayram E, Atalay C, Altas E, et al. Neurogenic stress cardiomyopathy following subarachnoid hemorrhage is associated with vagal complex degeneration: first experimental study. World Neurosurg. 2019;129:e741-e748.

25. Endemann DH, Schiffrin EL. Endothelial dysfunction. J Am Soc Nephrol. 2004;15(8):1983-1992.

26. Furchgott RF, Zawadzki JV. The obligatory role of endothelial cells in the relaxation of arterial smooth muscle by acetylcholine. Nature. 1980;288(5789):373-376. 